\title{
Dynamic Modeling and Simulation of Mechanical System Based on Dynamic Test Data
}

\author{
Huabing Chen \\ \{ chenhuapinzz@163.com\} \\ Sichuan Electric Vocational and Technical College Chengdu 611133, China
}

\begin{abstract}
In this paper, a dynamic model of mechanical system based on experimental data is proposed by combining the physical simulation and computer simulation. System level model updating is a key issue in modern mechanical design, such as flexible multibody system dynamics modeling, mechanism dynamics modeling and joint modeling and so on. In this paper, the dynamic simulation of mechanical system based on dynamic experimental data is carried out, which is based on the two aspects of the vibration problem and the dynamic balance of mechanism. For the mechanical system based on vibration, the simulation results are close to the experimental results by modifying the modal damping ratio of the components and the parameters of the flexible joint. For institutions comprehensive dynamic balance problem by means of computer simulation, and designed an optimization process for this kind of problems. It also uses the contrast experiment to modify the structural modal damping ratio, respectively, to verify the effectiveness of optimization and the method of correction. The mechanical system dynamics analysis, the first step is to build up the basic model of flexible multibody systems, in order to obtain better results, but also need to further realize the mechanical system dynamics pre correction model then by digital simulation to guide the design finally from the experimental data according to the starting, through comparison of experiment and simulation, the important parameters of the system level model modified, so as to establish the digital simulation model based on experimental data. By comparison with the experiment shows that, the method in this paper can not only trend prediction effect, also can obtain more accurate simulation results, from the experimental and simulation combined with this point of view to explore the mechanical system dynamics simulation analysis method.
\end{abstract}

Keywords: Model updating; flexible multibody system; flexible joint; dynamic balance; simulation and optimization

\section{Introduction}

High speed, high efficiency and load quality than large, sophisticated mechanical system has become a $\mathrm{R} \& \mathrm{D}$, production of the mainstream of modern machine tools, the dynamic performance of machine tool products is affecting their work performance, an important indicator of the quality of product life and, the configuration of the actual machine tool system is by a limited number of institutions and structures by kinematic pairs connecting open and closed loop or a variable structure of multibody system acquisition. How to achieve rapid and effective product design and technology update, the rapid occupation of the product market, 
improve their competitiveness has become the completion of the machine tool system design and simulation analysis of the problem to be solved. Therefore, due to the rapid development of fierce competition in the market pull and technology push interaction, oriented to the hierarchical system of modern design and simulation method of Virtual Prototype Collaborative Design and simulation technology came into being, modern product design method has from the oriented parts of the traditional static design, design experience and analogy design method gradually transformed for system level dynamic coordination and optimization design method. Very weak vibration of the machine tool has the potential to affect the precision or ultra precision machining accuracy. Because of high flexibility, high precision, high speed, high efficiency and high reliability [1], the high speed or quasi high speed machining spindle has become more and more important. With the improvement of machining precision of parts and the rapid increase of product output, it is required to develop in high precision, Gao Gang degree and high speed, and the dynamic performance of the machine is higher and higher. Because the dynamic performance of the whole machine is decided by its various components, the spindle system is one of the most important parts of the machine tool. High speed or quasi high speed spindle unit is the direct power of the whole machine tool system to provide power and processing components, so the whole system is the most important. In the front end of the spindle mounting tool or workpiece, directly involved in machining, the machining accuracy of machine tools, in the mechanical power or rotating machinery and transmission system or transmission shaft as important parts, its function is the motor output torque power effectively passed to the actuator, are widely used in machine tools, turbines and other common or special machinery. The static and dynamic performance directly affects the performance of the whole system to. From the energy point of view, the energy of the machine tool vibration comes from the interaction between the machine tool drive system, the components and the main shaft system, including the forced vibration and the. To the machine vibration, the influence of the spindle system accounts for a large proportion, and the spindle system is the end point of the transmission path of the power of the machine tool, and its dynamic response directly determines the dynamic performance of the machine tool system.

\section{Multi Body Dynamics Modeling and Simulation of Rigid Flexible Coupling Method}

In the complex mechanical system, the flexible parts of the elastic deformation of large deformation and small deformation of the rigid flexible coupling multibody system dynamics equations showing a high nonlinearity and different displacement modes between strong coupling characteristics, increase the degree of freedom of the system [2], complicate the components of kinematic and dynamic relations. Because of the nonlinear and high dimension, numerical simulation calculation as the analysis of rigid flexible coupling together the effective method of multibody system dynamics is an indispensable part of, so until the 1980s in the computer technology, rigid flexible coupling synthetic analysis of multi body system dynamics really realize and rapid development. The flexible effect of the components in the multibody system is firstly concerned by many scholars in the field of aerospace and high speed aircraft. And multi rigid body system dynamics similar to that of rigid flexible coupling synthetic modeling method for multibody system dynamics also formed two different ways of describing. The main difference between the two is the generalized coordinates 
contains a description of the flexible deformation of the elastic coordinates, namely Lagrangian coordinates and elastic coordinates relative description method and the Cartesian coordinates and elastic coordinates of absolute description method.

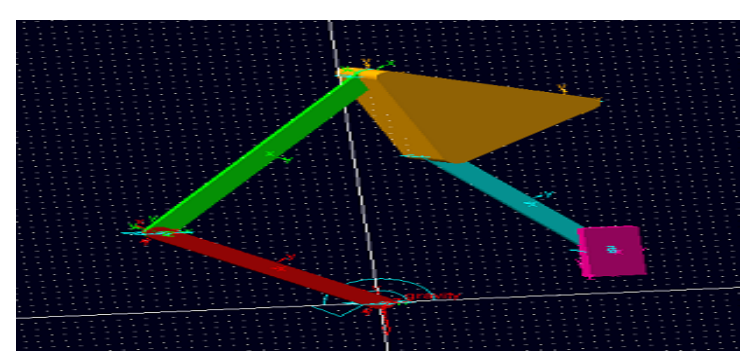

Fig. 1. Three dimensional model of crank slider mechanism

\subsection{Modified Modal Integration}

High speed machining or quasi high speed machining requires that the machine system has a good dynamic characteristics. In order to meet the requirements of modern productivity, precision and reliability, however, in practical engineering, machining performance of machine tool system in a large extent depending on the dynamics of the spindle system response characteristics and reliability. Finite element method and modal test method are used to study the most extensive static or quasi-static elastic dynamic methods of the spindle system. However, these methods are difficult to deal with the dynamic and dynamic problems such as the dynamic and dynamic problems of the coupling between the multi body parts, such as the large range of the whole motion and the nonlinear dynamic response of the friction contact. And the rigid flexible coupling multibody dynamics can better deal with large displacement and small deformation coupling problem, can effectively solve the problem of kinematics and dynamics finite element method and modal test method cannot handle [3]. In addition to considering the large displacement constraints and problems encountered in the analysis of multi rigid body system dynamics, flexible body modeling and deformation movement and the overall rigid motion between the mutual coupling puly rigid flexible coupling multibody system dynamics research core problem, that is, how to flexible effect is introduced into the dynamic control equation. Therefore, rigid flexible coupling method of spindle system rigid flexible coupling multibody dynamics behavior is practical function need to solve the problem.

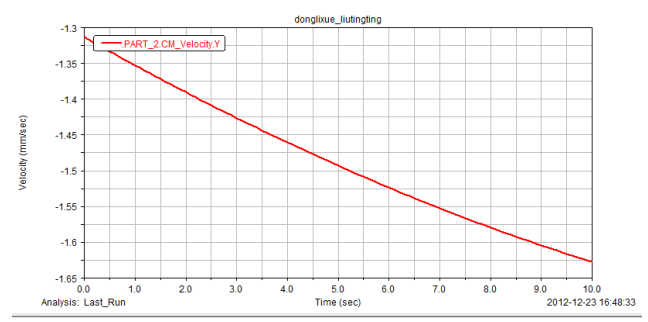


Fig. 2. Y direction movement speed curve of parts

\subsection{Implementation of Collaborative Simulation}

Collaborative design and simulation is remote and distributed modeling and simulation analysis of personnel in a collaboration, interoperability environment convenient, fast and friendly use in their respective fields of professional analysis tools, to constitute a system of each subsystem were modeling and simulation analysis, or from different views of function, performance of single point analysis, and transparent support for them to participate in the joint simulation of the whole system and collaboration to complete the system design and Simulation of a complex system design and simulation analysis method. Therefore, the data exchange always runs through the whole process of collaborative design and simulation, and all kinds of virtual product model and the different people in the form of data flow to complete the complex system of virtual product development. Product function design and simulation analysis of the special system of oriented, such as machine tool product development design and simulation analysis of relates to multi-body system kinematics, dynamics, combination of static and dynamic characteristics, static and dynamic analysis and optimization design, vibration and noise and hydraulic control characteristics or field, to consider the machine, electricity, liquid, control a multi-body model of disaster synthetic characteristics or field to establish a unified virtual prototype model is necessary [4]. The whole life cycle, Product Virtual Prototype Collaborative Design and simulation is inevitably involved in the data exchange between all kings and other digital model, using mature software of virtual product system for data exchange must clear product virtual design and simulation analysis of the purpose, because different purposes will determine the different data exchange format, should compare the advantages and disadvantages of all kinds of software, such as software compatibility and validity of data to ensure system of the virtual product model for parametric modeling, simulation analysis, model modification and optimization design and control etc. results the correctness.

\section{Simulation and Implementation of the System}

Systematically introduce description and choice of coordinates of multi body system dynamics, based on the choice of generalized coordinates, the rigid flexible coupling synthetic multibody system dynamics equations are deduced, and improve the second chapter elaboration component modal disaster legal theoretical description. The velocity and acceleration equations of any point on the flexible point are derived, and the Euler parameters are used to describe the spatial orientation of the flexible, to simplify the equations of velocity and acceleration. Finally using the first kind of Lagrange multiplier equation of the rigid flexible coupling multibody system dynamics equations, and discuss the multi body system of common physical rigidity and numerical stiffness and method of selecting solver. In highspeed rotation of the spindle system, the flexible deformation of main shaft and bevel gear to the dynamic performance of the system have great influence, such as system of disaster combined frequency and dynamic displacement etc.. Therefore, this paper mainly adopts establishing collaborative design and simulation platform and the chapter of rigid flexible coupling multibody system dynamics theory, completion of main rigid flexible coupling joint establishment and dynamics analysis of multibody system dynamics model, study speed and 
cutting excitation force of system dynamic characteristics. The displacement response of the radial vibration is much larger than that obtained by the quasi static finite element method in the reference literature. The displacement amplitude of the forced vibration is obtained. This shows that the machine tool spindle failed to resonate, in under the combined action of the cutting force and speed incentives, the radial vibration displacement response is one of the main reasons for its dynamic characteristics and processing performance, namely the radial vibration of flexible tool spindle end node directly affects the machining accuracy and surface quality of spindle system dynamic performance and milling parts. The analysis results also illustrate the spindle speed and cutting incentive is one of the main factors that influence the dynamic displacement of the spindle bearing components, also confirmed that the literature review in ignoring dynamic incentive factors or in the quasi static method, the equivalent dynamic excitation of traditional methods of analysis result has a certain error [5].

\section{Conclusion}

High speed machining or quasi high speed machining requires that the machine system has a good dynamic characteristics. In order to meet the requirements of modern productivity, precision and reliability, however, in practical engineering, machining performance of machine tool system in a large extent depending on the dynamics of the spindle system response characteristics and reliability. Combined with the experimental data of the spindle components, the simulation model of the spindle bearing is completed, and the forced vibration characteristics of the flexible model of the main spindle bearing and the dynamic characteristics of the boundary conditions are analyzed. According to the acceptance test of milling machine of the boundary conditions, analysis of different cutting depth value and different speed of rigid and flexible coupled synthetic effect of the dynamic characteristics of the spindle system.

\section{References}

[1] Deng Fengyan, and Xing lock, Zhang Juan, Li Liang, Zhang Liexia. Application of the modified Craig-Bampton method in multibody system dynamics modeling. No. 03. (2014)

[2] Wang Jianming, Hong Jiazhen, Liu Youwu. A new modeling method for rigid flexible coupling system. Journal of vibration engineering. No. 02. (2013)

[3] Yang Hui, Hong Jiazhen, Yu Zhengyue. Experimental verification of the modeling theory of rigid flexible coupling. Journal of mechanics, No. 02. (2013)

[4] Yan Shaoze, Shen Yongsheng, Chen Hongbin. Numerical simulation of the deployable structure dynamics of the flexible and hinged joints. Journal of Tsinghua University (Natural Science Edition). No. 02. (2013)

[5] Xu Zhang Ming, Shen Rongying, Hua is found. Based on the sensitivity of the frequency response function of the correlation analysis of finite element model updating. Journal of mechanical strength. No. 01. (2013) 